\title{
Energy-Saving Method of Parallel Mechanism by Redundant Actuation
}

\author{
Giuk Lee',", Seung-Ki Sul', and Jongwon Kim \\ 1 School of Mechanical and Aerospace Engineering, Seoul National University, 1, Gwanak-ro, Gwanak-gu, Seoul, 08826, South Korea \\ 2 School Electrical and Computer Engineering, Seoul National University, 1, Gwanak-ro, Gwanak-gu, Seoul, 08826, South Korea \\ \# Corresponding Author / E-mail: giuk.lee87@gmail.com, TEL: +82-2-880-7144, FAX: +82-2-875-4848
}

KEYWORDS: Parallel machines, Redundant actuation, Energy efficiency, Manipulators

This paper presents a novel energy-saving method of a robotic system utilizing parallel mechanism by redundant actuation. The redundantly actuated system can distribute arbitrary actuating torques in a certain combination. By using this feature, the regenerative power dissipation and electric power loss are reduced. Moreover, the redundant actuation can reduce the peak torque of actuating joints. This feature reduces the friction loss because we can use a smaller gear reducer. A parallel manipulator with two degrees of freedom is simulated as a case study. The energy consumption of the robotic system is modeled by analyzing the type of energy consumption in actuation systems. An optimization process is proposed to maximize the energy-saving effect. The results show that $26.1 \%$ of electric energy can be saved by redundant actuation.

Manuscript received: February 27, 2015 / Revised: May 4, 2015 / Accepted: July 21, 2015

\section{NOMENCLATURE}

$\tau=$ Load torque at the actuating joint

$\tau_{u}=$ Load torque vector of the non-redundant actuation

$\tau_{r}=$ Load torque vector of the redundant actuation

$\tau_{f}=$ Friction torque in the harmonic drive

$\tau_{m}=$ Load torque at the motor

$q_{u}, q_{v}=$ Angle vectors of the independent and dependent joint of non-redundant actuation, respectively

$q_{r}=$ Angle vector of the actuating joint of redundant actuation

$\dot{q}=$ Rotation velocity of the actuating joint

$\dot{q}_{m}=$ Rotation velocity of the motor

$\Gamma=$ Jacobian mapping from the joint velocity vector of nonredundant actuation to that of redundant actuation

$\mathrm{U}=$ Transfer matrix from the independent and dependent joint vectors to the whole joint vectors

$\mathrm{V}=$ Selection matrix for the actuating joints from the whole joint vectors

\section{Introduction}

The energy efficiency of a robotic system is considered an important issue in the academic and industrial fields. ${ }^{1-3}$ A great deal of energy is dissipated as heat loss during the actuation of a robotic system. The energy inefficiency occurs this energy loss. Therefore, the reduction of energy loss can improve energy efficiency.

Various studies have been conducted to try to solve this problem. Recently, Endo et al. developed a gravity compensation mechanism with a non-circular pulley and a spring. ${ }^{4-6}$ Khoukhi et al. studied the constrained multi-objective trajectory planning of a parallel mechanism by considering the actuator dynamics to improve energy efficiency. ${ }^{7,8}$ On the side of electric devices, Seok et al. installed a regenerative system in a Massachusetts Institute of Technology (MIT) cheetah robot to reuse regenerative power. ${ }^{9}$

In this paper, we present novel energy-saving method of a robotic system utilizing parallel mechanism by redundant actuation. The redundant actuation can distribute arbitrary actuating torques in a certain combination. Lee et al. show that electric power loss can be reduced by this feature. ${ }^{10,11}$ We firstly show that not only electric power loss but also regenerative power dissipation and friction loss can be reduced by redundant actuation. A simulation is performed to show the possibility of the energy-saving method using the optimal torque distribution of redundant actuation. 
The energy-saving concept by redundant actuation is presented in Section II. The simulation of a parallel manipulator with two degrees of freedom $(\mathrm{DoF})$ is performed as a case study to verify the energy-saving effect. The kinematic and dynamic models and the test condition is presented in Section III. The energy consumption of the actuator is analyzed and modeled according to type in Section IV. In Section V, the optimization algorithm for minimizing the energy consumption and deriving the optimal torques is proposed, and the simulation result of optimization is presented. Finally, the concluding remarks follow in Section VI.

\section{Concept}

Redundant actuation means that a robotic system uses more actuators than DoF. In this case, the $\tau_{r}$ is related to the $\tau_{u}$ of the same robotic system in Eq. (1) according to the principle of virtual work

$$
\begin{gathered}
\tau_{u}^{T} \dot{q}_{u}=\tau_{r}^{T} \dot{q}_{r}=\tau_{r}^{T}\left(\Gamma \dot{q}_{u}\right) \\
\tau_{u}=\Gamma^{T} \tau_{r}
\end{gathered}
$$

From this relationship, the actuating torque $\tau_{r}$ has a null space solution. We can determine the actuating torque in a certain combination from Eq. (2). ${ }^{12}$

$$
\tau_{r}=\left(\Gamma^{T}\right)^{+} \tau_{u}+\left(I_{n}-\left(\Gamma^{T}\right)^{+} \Gamma^{T}\right) \varepsilon_{n}
$$

where $(\bullet)^{+}$is the generalized inverse of the pseudo inverse; and $\varepsilon_{n}$ is an arbitrary vector for determining the magnitude of the null space vector, which is called the internal torque.

By using this torque distribution feature, we can assign the optimal actuating torques to minimize the energy loss of the system. It can be realized by the reduction of regenerative power dissipation, electric power loss, and friction loss.

\subsection{Regenerative Power Dissipation Reduction}

Regenerative power occurs in motors owing to externally supplied power when the motion and force direction of the actuator are opposed. This is called negative work. The regenerative power can be converted to an available power source by the regenerative system. ${ }^{9}$ However, this technology is very expensive to construct; therefore, the regenerative power is usually dissipated as heat by the internal dump resistor. This causes energy loss.

Let us consider two types of a 2-DoF parallel manipulator operating with a $30-\mathrm{kg}$ payload according to the same pathway. In the case of a 2-DoF non-redundant manipulator, as shown in Fig. 1(a), negative work occurs. In this pathway, motor 2 has to produce the actuating torque in a clockwise direction to sustain the load; however, it has to rotate counterclockwise because of the kinematic constraint of the manipulator. In addition, motor 1 must produce more positive work than the mechanical energy required by the manipulator, owing to the principle of virtual work. As a result, more output power equal to the absolute value of negative work should be produced to make up for the unnecessary negative work of the motors.

If the manipulator of Fig. 1(a) is extended to redundant actuation as shown in Fig. 1(b), the negative work of motor 2 can be eliminated by the optimal distribution of actuating torques. In this case, the actuating

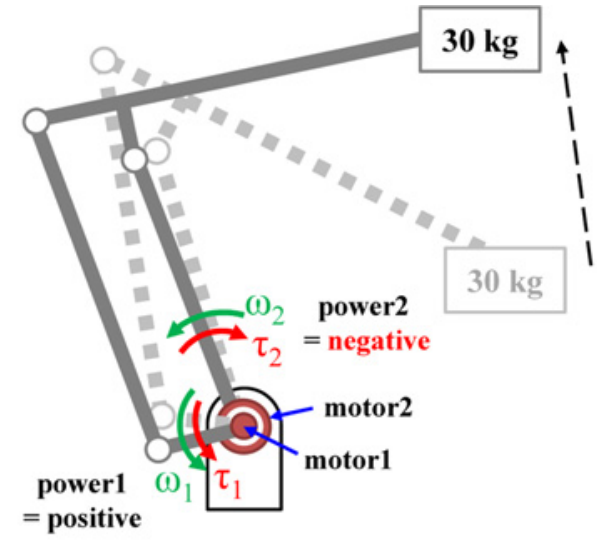

(a) Negative work in 2-DoF non-redundant manipulator

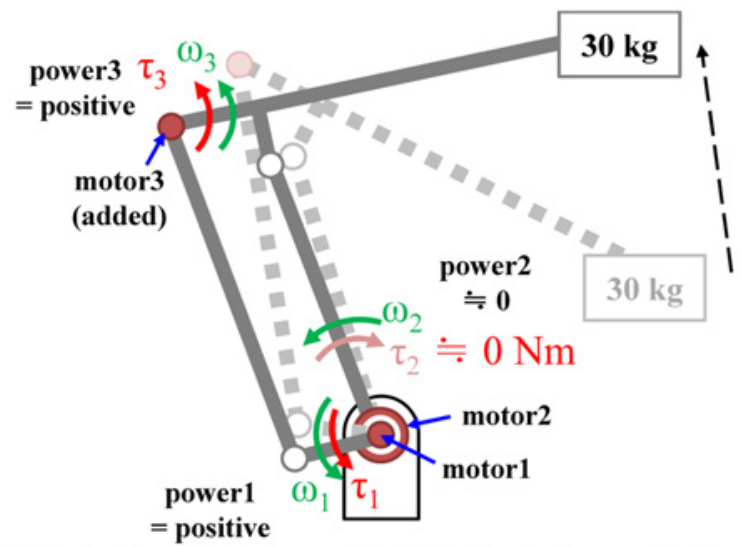

(b) Reduction of negative work in 2-DoF redundant manipulator

Fig. 1 Reduction of negative work by extension to redundant actuation: regenerative power dissipation reduction

torque of motor3 sustains the payload instead of motor2, and motor2 does not have to do negative work. This leads to the reduction of the required output power of the overall motors. By keeping this concept in mind, we can reduce the regenerative power dissipation by negative work in the same pathway.

\subsection{Electric Power Loss Reduction}

The input energy for actuating a robotic system is not only used for output power but is also consumed as electric power loss. In this study, we consider the copper loss of the motor, which are known as the main causes of electric power loss. Electric power loss occurs in proportion to the square of the current and is dissipated as heat.

The required motor current is known to be proportional to the required torque. Therefore, the torque distribution of redundant actuation can distribute the current. When we distribute the torques to minimize the sum of square torques $\left(\sum \tau^{2}\right)$, the total electric power loss can be reduced.

In Fig. 2, two types of 2-DoF manipulators are holding a 30-kg payload at a constant position. The non-redundant manipulator in Fig. 2(a) produces $322,600(\mathrm{Nm})^{2}$ for $\sum \tau^{2}$. When we extend this manipulator to redundant actuation, as shown in Fig. 2(b), and optimally distribute the actuating torques to minimize $\sum \tau^{2}$, the value is reduced to $102,900(\mathrm{Nm})^{2}$. As a result, the electric power loss can be reduced by $68 \%$ at the same posture. This is the concept of electric power loss reduction. 


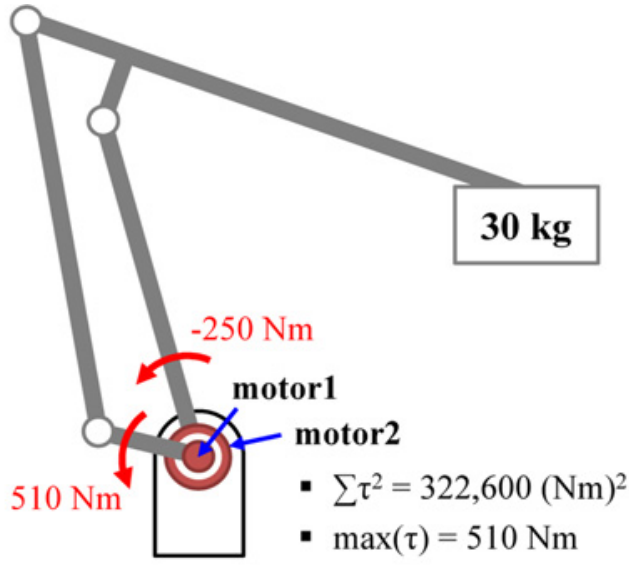

(a) Sum of square torques and peak torque in 2-DoF non-redundant manipulator

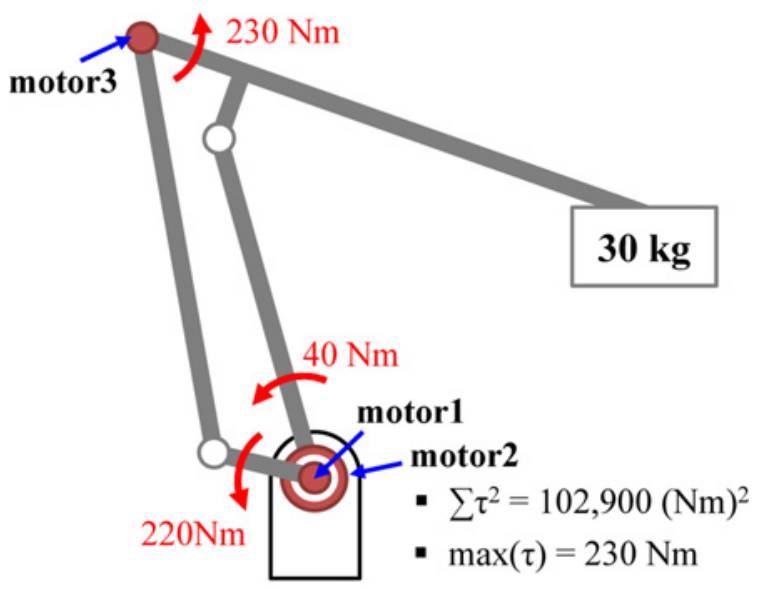

(b) Sum of square torques and peak torque in 2-DoF redundant manipulator

Fig. 2 Reduction in sum of square torques and peak torque by extension to redundant actuation: electric power loss and friction loss reduction

\subsection{Friction Loss Reduction}

The manipulators use the gear reducer to increase the torque capacity of the actuating joint. When the actuating joint rotates, the friction loss occurs in the reducer owing to its friction torque such as coulomb and viscous friction. As the size of the reducer is increased, the friction loss is increased owing to the increased friction torque.

In the case of the non-redundant actuation, as shown in Fig. 2(a), the required actuating torques are high because of the high condition number of the posture, which indicates the nearby actuator singularity region. These high torques increase the required specification of the motor and gear reducer because their torque capacity should be selected based on the peak torque.

The peak torques can be reduced when the manipulator is extended to redundant actuation, as shown in Fig. 2(b). This can be realized by the reduction of the condition number because we can distribute the required torque by the additional motor, which produces a new actuating torque vector. ${ }^{13}$ In the case of Fig. 2, the peak torque is reduced by 55\% from $510 \mathrm{Nm}$ to $230 \mathrm{Nm}$ by redundant actuation. This can reduce the friction loss of each actuating joint because the friction torque is reduced by the smaller gear reducer. Additionally, despite the additional actuator, the overall friction loss can be reduced.

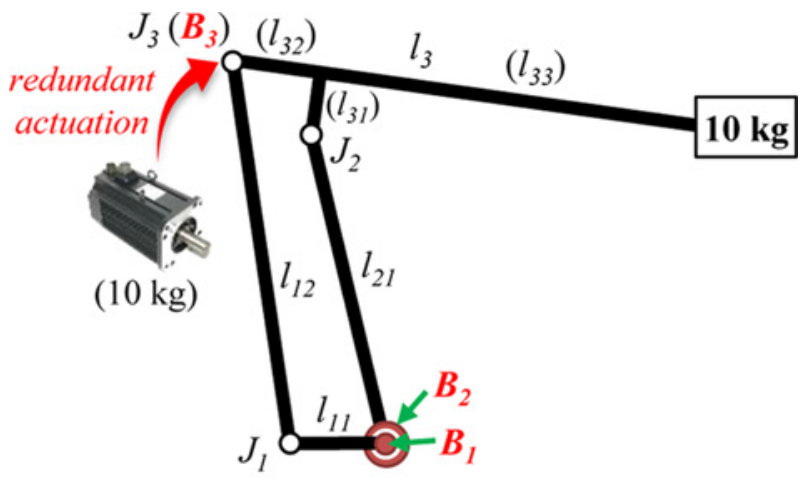

(a) Schematic of 2-DoF test manipulator

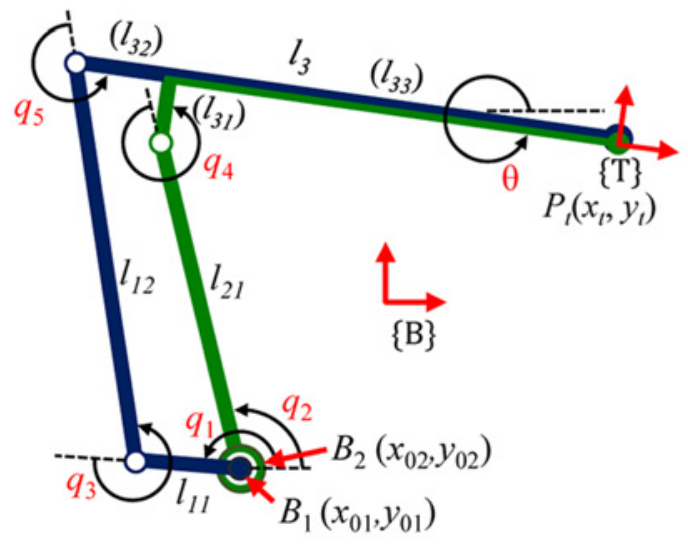

(a) Notation of 2-DoF test manipulator

Fig. 3 Schematic and notation of 2-DoF test manipulator

Table 1 Specifications of 2-DoF test manipulator

\begin{tabular}{cccc}
\hline Part & Length $(\mathrm{mm})$ & Weight $(\mathrm{kg})$ & Moment of inertia $\left(\mathrm{kg} \cdot \mathrm{m}^{2}\right)$ \\
\hline$l_{11}$ & 230 & 3.7 & 0.07 \\
\hline$l_{12}$ & 960 & 3.7 & 1.21 \\
\hline$l_{21}$ & 800 & 16 & 2.61 \\
\hline$l_{3}$ & 1310 & 21 & 6.99 \\
\hline
\end{tabular}

\section{Case Study: 2-DoF Parallel Manipulator}

\subsection{Kinematic and Dynamic Models}

A parallel-type 2-DoF manipulator is used to simulate the energysaving effect by redundant actuation. Fig. 3(a) shows a schematic of the test manipulator. The upper linkage $l_{3}$ is connected by two chains: $l_{11}$ and $l_{12}$, and $l_{21}$. Two motors are installed at $B_{1}$ and $B_{2}$ for nonredundant actuation. For redundant actuation, an additional actuator is installed at $B_{3}$, which is labeled $J_{3}$ in the non-redundant actuation case. The weight of the additional actuator, including a motor and reducer, is assumed as $10 \mathrm{~kg}$. This manipulator is based on the main link mechanism of a commercial manipulator (IRB1410, ABB, Zurich, Switzerland). The detailed specifications of the manipulator are listed in Table 1.

The kinematic model for the proposed test manipulator can be derived from the constraint equation. The ends of two serial chains, which are marked as blue and green in Fig. 3(b), are coincident at one point. Assuming that the end coordinates of the $i$-th chain are $P_{t i}\left(x_{t i}, y_{t i}\right)$, the geometrical constraints of the mechanism are shown in the following equation: 


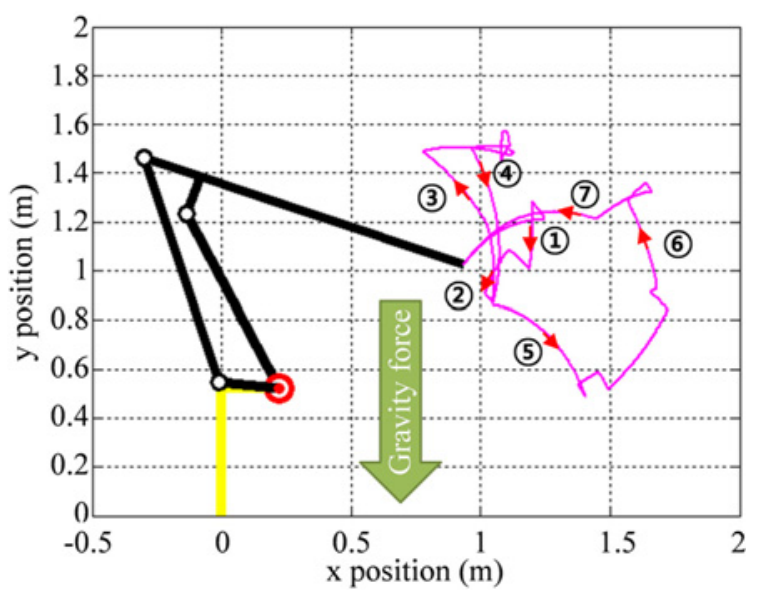

Fig. 4 Test pathway of 2-DoF test manipulator

$$
\begin{gathered}
g\left(q_{a l l}\right)=0:\left[\begin{array}{l}
g_{1} \\
g_{2} \\
g_{3}
\end{array}\right]=\left[\begin{array}{c}
x_{t 1}-x_{t 2} \\
y_{t 1}-y_{t 2} \\
\phi_{1}-\phi_{2}+\frac{\pi}{2}
\end{array}\right]=\left[\begin{array}{l}
0 \\
0 \\
0
\end{array}\right] \\
\phi_{1}=q_{1}+q_{3}+q_{5}=\theta, \phi_{2}=q_{2}+q_{4}=\theta+\frac{\pi}{2}
\end{gathered}
$$

where the whole joint vector $q_{\text {all }}=\left[q_{1} q_{2} q_{3} q_{4} q_{5}\right]^{\mathrm{T}}$ and $\theta$ is the rotation angle of the end effector frame. The velocity relationship between the joint angles is calculated by the time derivative of the geometric constraint as Eq. (4). ${ }^{13}$

$$
\frac{d g}{d t}=\frac{\partial g}{\partial q_{u}} \frac{d q_{u}}{d t}=\frac{\partial g}{\partial q_{v}} \frac{d q_{v}}{d t}=\left[G_{u} G_{v}\left[\begin{array}{l}
\dot{q}_{u} \\
\dot{q}_{v}
\end{array}\right]=0\right.
$$

where $q_{u}=\left[q_{1} q_{2}\right]$ and $q_{u}=\left[q_{3} q_{4} q_{5}\right]$. Using this equation, the Jacobian mapping matrix $\Gamma$ can be derived as Eq. (5)

$$
\dot{q}_{r}=V \dot{q}_{\text {all }}=V U\left[\begin{array}{c}
I_{2 \times 2} \\
-G_{v}^{-1} G_{u}
\end{array}\right] \dot{q}_{u}=\Gamma \dot{q}_{u}
$$

where $q_{v}=\left[\begin{array}{lll}q_{1} & q_{2} & q_{5}\end{array}\right]$.

The dynamic model of a redundantly actuated manipulator can be derived by replacing that of the non-redundantly actuated manipulator with the constraint Eq. (1). This is shown in the following equation:

$$
M(q) \ddot{q}_{u}+C(q, \dot{q}) \dot{q}_{u}+N(q)=\tau_{u}=\Gamma^{T} \tau_{r}
$$

where a $10-\mathrm{kg}$ payload at the end effector is included in the dynamic model to consider the effect of the working unit's weight. The weight of the additional actuator was only considered when we analyzed the dynamic model of the redundantly actuated manipulator.

\subsection{Test Condition}

The test pathway is shown in Fig. 4. It starts at Position 1 and finishes at Position 7. We determined this by referencing the spot welding pathway, which is provided from the Hyundai automotive company. The overall actuating time is $39 \mathrm{~s}$, and the maximum velocity of the end effector is $480 \mathrm{~mm} / \mathrm{s}$.

The simulation is performed by comparing the energy consumptions
Table 2 Specification of motor and harmonic drive for non-redundant and redundant actuation

\begin{tabular}{cccc}
\hline & & Non-Redundant & Redundant \\
\hline \multirow{3}{*}{ Motor } & Rated torque & $3.5 \mathrm{Nm}(2 \mathrm{EA})$ & $2 \mathrm{Nm}(3 \mathrm{EA})$ \\
\cline { 2 - 4 } & Rotation speed & $1000 \mathrm{rpm}$ & $1000 \mathrm{rpm}$ \\
\cline { 2 - 4 } & Rated power & $350 \mathrm{~W}$ & $200 \mathrm{~W}$ \\
\hline \multirow{2}{*}{$\begin{array}{c}\text { Gear } \\
\text { reducer }\end{array}$} & Product number & CSF40160 & CSF50160 \\
\cline { 2 - 4 } & Reduction ratio & $1: 160$ & $1: 160$ \\
\cline { 2 - 4 } & Rated torque & $529 \mathrm{Nm}$ & $294 \mathrm{Nm}$ \\
\hline
\end{tabular}

of non-redundant and redundant actuation in the same pathway. The energy-saving effect is calculated by the energy consumption model, which will be analyzed in Section IV.

Table 2 shows the selection results of the motor and gear reducer according to the pre-simulation. The harmonic drives are generally known as the most commonly used gear reducer in the industrial manipulators. Therefore, we select the harmonic drive as the gear reducer.

As described in Section II, the redundant actuation can reduce the required rated power of the motor and the size of the harmonic drive. In addition, the reduction of the size of the harmonic drive can lead to friction loss reduction, although its reduction rate is not reduced.

\section{Energy Consumption Analysis}

\subsection{Mechanical Power of Motor}

Mechanical power is calculated by the production of load torque and the rotation velocity of the motor. In the case of negative output power, power for the motor is supplied externally and dissipates as heat because the motor usually cannot regenerate that power but instead loses it. Therefore, the mechanical power of the motor can be expressed as Eq. (7)

$$
P_{\text {mech }}=\left[\tau_{m} \dot{q}_{m}\right]^{+}
$$

where $[x]^{+}$means that if $x$ is positive, $[x]$ is $x$; otherwise, $[x]$ is zero.

\subsection{Electric Power Loss}

Electric power loss mainly occurs by the copper loss $P_{\text {copper }}$, and it can be calculated as Eq. (8).

$$
P_{\text {copper }}=R I^{2}
$$

where $R$ is the internal resistance of motors, and $I$ is the flow current.

The value of copper loss varies according to the type of motor. Even with the same type of motor, it is affected by operating conditions such as the temperature. Therefore, it is difficult to determine the exact value of the copper loss in the simulation. When the state of the art motor is operating at the rated power, the energy efficiency is known to amount to approximately $90 \%$. We assume the other $10 \%$ to be the electric power loss due to the copper loss.

Therefore, the electric power loss $P_{\text {elec }}$ can be expressed as Eq. (9).

$$
P_{\text {elec }}=P_{\text {elec }}^{\text {rated }}\left(\frac{\tau_{m}}{\tau_{m}^{\text {rated }}}\right)^{2}\left(P_{\text {elec }}^{\text {rated }}=0.10 \times P_{\text {rated }}\right)
$$

where $P_{\text {rated }}$ is the rated power of the motor, and $\tau_{m}^{\text {rated }}$ is the rated torque of the motor. 


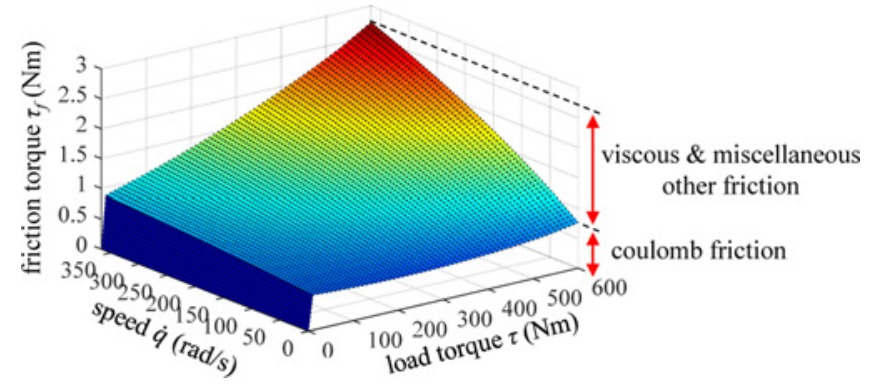

Fig. 5 Friction torque of harmonic drive (Harmonic Drive, CSF50160, MA, USA) according to load torque and rotation speed

\subsection{Friction Loss}

Friction loss mainly arises from the gear reducer of the manipulator. We selected the harmonic drive as the gear reducer of the case study model. The friction torque can be modeled based on several effectse.g., coulomb, viscosity friction. ${ }^{14}$ But we derive the trend equation based on the product catalog of the harmonic drive for a more credible equation. ${ }^{15}$ The trend equation is shown in Eq. (10).

$$
\begin{gathered}
\tau_{f}=\frac{(1-\eta) \tau}{\eta N} \operatorname{sign}\left(\dot{q}_{m}\right) \quad(0<\eta<1) \\
\eta=\left(\frac{a_{1} x^{2}+a_{2} x+a_{3}}{x+a_{4}}\right)\left(\frac{b_{1} y+b_{2}}{y+b_{3}}\right), x=\frac{\tau}{\tau_{h}^{\text {rated }}}, y=\left|\dot{q}_{m}\left(\frac{60}{2 \pi}\right)\right|
\end{gathered}
$$

where the efficiency $\eta$ is calculated by Eq. (11), $\tau_{h}^{\text {rated }}$ and $N$ are the rated torque and the reduction ratio of the harmonic drive, respectively.

As a case study, the experimental friction torque of a harmonic drive (CSF50160, Harmonic Drive, MA, USA) is shown in Fig. 5. We obtained the experimental data from the product catalog. ${ }^{12}$ It consists of the coulomb, the viscous, and the miscellaneous other friction types. As the rotation speed and load torque are increased, the friction torque is increased. The values of the constants are as follows: $\left(a_{1}=-1.290 \times 10^{-1}\right.$, $a_{2}=1.325, a_{3}=1.135 \times 10^{-4}, a_{4}=1.981 \times 10^{-1}, b_{1}=3.239 \times 10^{-1}, b_{2}=2.961$ $\times 10^{3}$, and $\left.b_{3}=3.558 \times 10^{3}\right)$.

\subsection{Power Consumption Model}

The power consumption model of an actuator $P_{a c t}$ can be expressed as Eq. (12).

$$
\begin{gathered}
P_{\text {act }}=P_{\text {mech }}+P_{\text {elec }}=\left[\tau_{m} \dot{q}_{m}\right]^{+}+P_{\text {elec }}^{\text {rated }}\left(\frac{\tau_{m}}{\tau_{m}^{\text {tated }}}\right)^{2} \\
\tau_{m}=\frac{\tau}{N}+\tau_{f}, \dot{q}=\frac{\dot{q}_{m}}{N}
\end{gathered}
$$

It can be substituted as a function of torque $\hat{o}$ and rotation velocity of the actuating joint in Eq. (14) by the relationship of torques and rotation velocities in Eq. (13).

$$
P_{\text {act }}=\left[\tau \dot{q}+N \tau_{f} \dot{q}\right]^{+}+P_{\text {elec }}^{\text {rated }}\left(\frac{\frac{\tau}{N}-\tau_{f}}{\tau_{m}^{\text {rated }}}\right)^{2}
$$

By using this model, we can calculate the power consumption of the actuator to the torque and rotation velocity.

\section{Optimization}

\subsection{Algorithm}

The power consumption model of a robotic system $P_{s y s}$ can be expressed as the sum of that of actuators. The energy consumption of a robotic system $E_{s y s}$ can be calculated by the integration of $P_{s y s}$ about actuating time as Eq. (15)

$$
\begin{gathered}
E_{\text {sys }}=\int_{t_{i}}^{t_{f}} P_{\text {sys }} d t=\int_{t_{i}}^{t_{f}} \sum_{i=1}^{n} P_{\text {act }}^{i} d t \\
=\int_{t_{i}}^{t_{f}} \sum_{i=1}^{n}\left\{\left[\tau_{i} \dot{q}_{i}+N_{i} \tau_{f i} \dot{q}_{i}\right]+{ }^{i} P_{\text {elec }}^{\text {rated }}\left(\frac{\frac{\tau_{i}}{N_{i}}-\tau_{f i}}{\tau_{m i}^{\text {rated }}}\right)^{2}\right\} d t
\end{gathered}
$$

where $\mathrm{n}$ is the number of the actuator; ${ }^{i} x$ and $x_{i}$ are the values of an $x$ variable of the $i$-th actuator; and $t_{i}$ and $t_{f}$ are the initial and final time of the pathway, respectively. Note that we consider the optimization of redundant actuation by optimal torque distribution in the same pathway as non-redundant actuation. Therefore the joint velocity vector is given by the pathway.

By minimizing the energy consumption $E_{s y s}$, the optimal actuating torque trajectory of a redundantly actuated robotic system can be obtained as the Eq. (16).

$$
\begin{array}{cc}
\underset{\tau_{r}(0)}{\operatorname{minimize}} & J\left(\tau_{r}\right)=E_{\text {sys }}\left(\tau_{r}\right) \\
\text { subject to } & \tau_{u}-\Gamma^{T} \tau_{r} \\
& \tau_{r}^{L} \leq \tau_{r} \leq \tau_{r}^{U}
\end{array}
$$

The minimizing process was executed by using a sequential quadratic programming (SQP) method. ${ }^{16}$ The SQP is one of the most successful methods for the numerical solution of constrained nonlinear optimization problems. The SQP method is a procedure that generates iterates converging to a solution of this problem by solving quadratic programs that are approximations of nonlinear optimization problems. The conditions of optimization are as follow: (Stopping criteria: $\left|J\left(\tau_{r}^{i}\right)-J\left(\tau_{r}^{i+1}\right)\right|<1 \times 10^{-4},\left\|\tau_{r}^{i}-\tau_{r}^{i+1}\right\|<1 \times 10^{-4}$, Boundary of control variable: $\tau_{r}^{L}=-300$, and $\tau_{r}^{U}=300$ )

\subsection{Simulation Result}

Figs. 6-8 show the torque of the actuating joint, the motor, and the friction for non-redundant and redundant actuation, respectively. The solid red line represents the actuation torque of $B_{1}$. The dotted blue line represents the actuation torque of $B_{2}$. The alternating long and short dashed green line represents the actuation torque of $B_{3}$. There are some vibrated torques due to the acceleration and deceleration at the spot welding point. As shown in these figures, the peak actuating torque and friction torque are reduced by redundant actuation.

The energy consumptions for non-redundant and redundant actuation are presented in Fig. 9. The red area is the required mechanical energy for the actuating robotic platform. The yellow area is the energy loss caused by friction loss. The green area is the energy loss caused by regenerative power dissipation. The blue area is the energy loss caused by electric power loss. The sum of the red, yellow, and green areas is the output energy of the motor, and the sum of all areas is the total energy consumption of the robotic platform. 


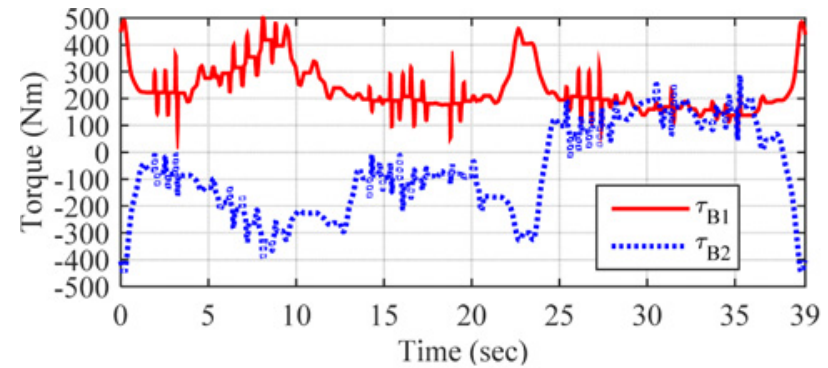

(a) Actuating joint torques of 2-DoF non-redundant manipulator

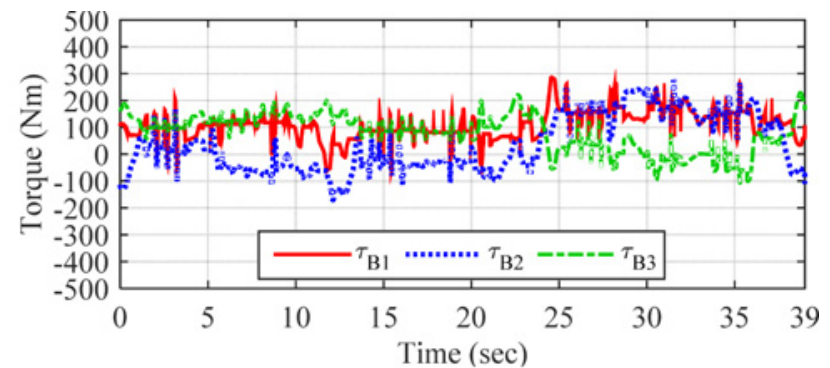

(b) Actuating joint torques of 2-DoF redundant manipulator

Fig. 6 Actuating joint torques of 2-DoF non-redundant and redundant manipulators

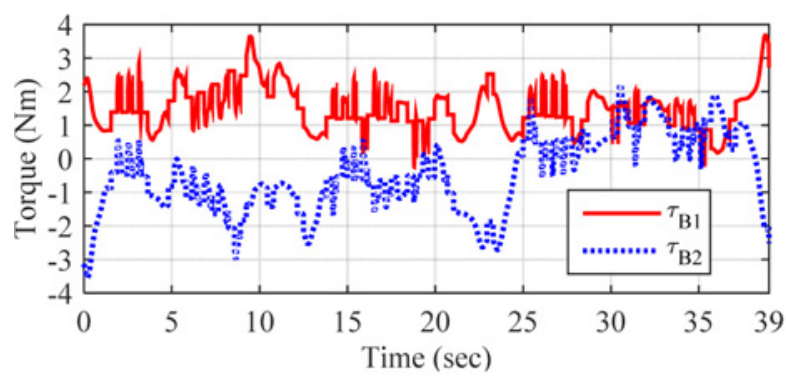

(a) Motor torques of 2-DoF non-redundant manipulator

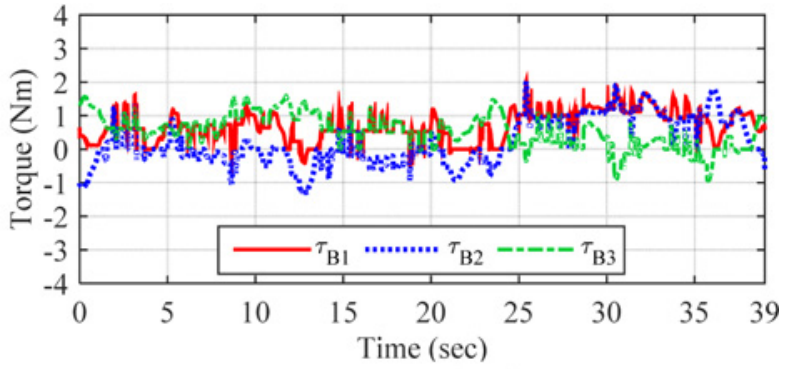

(b) Motor torques of 2-DoF redundant manipulator

Fig. 7 Motor torques of 2-DoF non-redundant and redundant manipulators

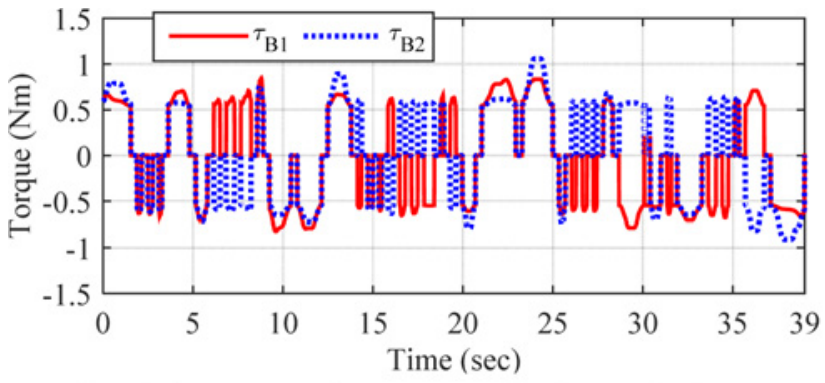

(a) Friction torques of 2-DoF non-redundant manipulator

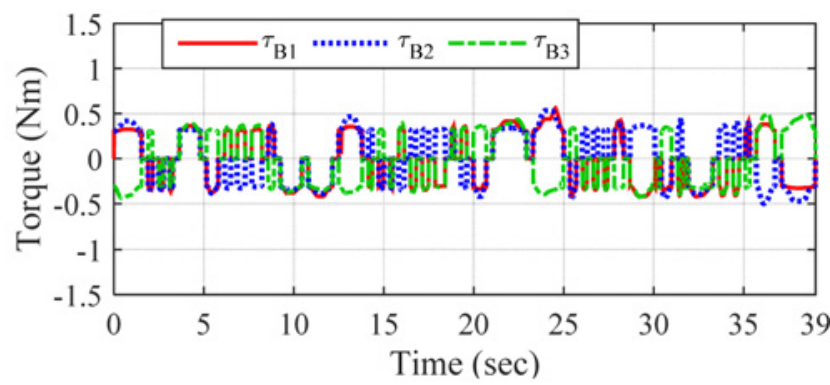

(b) Friction torques of 2-DoF non-redundant manipulator

Fig. 8 Friction torques of 2-DoF non-redundant and redundant manipulators

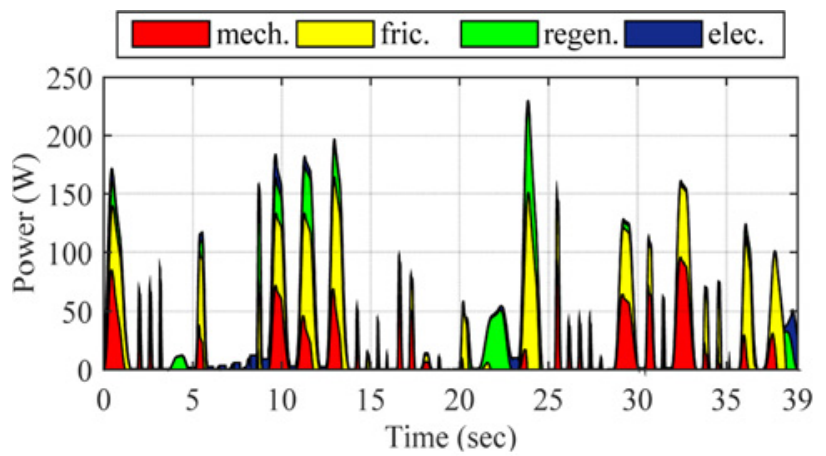

(a) Energy consumption of 2-DoF non-redundant manipulator

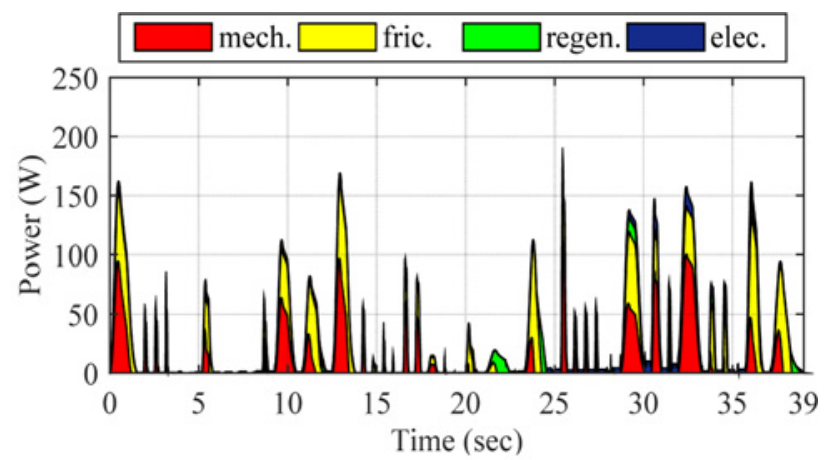

(b) Energy consumption of 2-DoF redundant manipulator

Fig. 9 Energy consumption of 2-DoF non-redundant and redundant manipulators

Table 3 Simulation of energy consumption

\begin{tabular}{ccccc}
\hline & & Non-Redundant & Redundant & Savings rate \\
\hline \multirow{2}{*}{$\begin{array}{c}\text { Output } \\
\text { energy } \\
\text { of motor }\end{array}$} & Mechanical work & $401 \mathrm{~J}$ & $410 \mathrm{~J}$ & $\boldsymbol{\Delta} 5 \mathrm{~J}(-2.2 \%)$ \\
\cline { 2 - 5 } & Regenerative power dissipation & $287 \mathrm{~J}$ & $71 \mathrm{~J}$ & $\boldsymbol{\nabla} 216 \mathrm{~J}(75.3 \%)$ \\
\hline & Friction loss & $692 \mathrm{~J}$ & $507 \mathrm{~J}$ & $\boldsymbol{\nabla} 185 \mathrm{~J}(26.7 \%)$ \\
\hline & Electric power loss & $135 \mathrm{~J}$ & $131 \mathrm{~J}$ & $\boldsymbol{\nabla} 4 \mathrm{~J}(3.0 \%)$ \\
\hline & Total energy consumption & $1,515 \mathrm{~J}$ & $1,120 \mathrm{~J}$ & $\boldsymbol{\nabla} 395 \mathrm{~J}(26.1 \%)$ \\
\hline
\end{tabular}


The values of energy consumption are arranged in Table 3 according to type. Note that the mechanical work increased in case of the redundantly actuated parallel manipulator due to the weight of additional actuator. The simulation results showed that energy consumption by redundant actuation was $26.1 \%$ less than that of nonredundant actuation.

\section{Conclusions}

We firstly present an energy-saving method of a parallel mechanism by redundant actuation. Savings are realized by the reduction of regenerative power dissipation, friction loss, and electric power loss. The optimization algorithm is presented to minimize the energy consumption by optimal torque distribution.

A 2-DoF parallel manipulator is simulated as a case study. The savings effect is calculated by comparing the energy consumption for a non-redundantly and redundantly actuated case of a parallel manipulator in the same pathway. The kinematic and dynamic models of two parallel manipulators are analyzed to simulate the saving effects. The simulation results show that $26.1 \%$ of energy can be saved by extension to the redundant actuation in the test pathway. We will verify these simulation results through experiments in the future.

The proposed energy-saving method can reduce the size of actuators; therefore, the volume of the robotic system is expected to be decreased. This feature is the additional benefit of the proposed method. The initial cost for redundant actuation using more actuators is not expected to be high because using more actuators can reduce the specification of each actuator-e.g., the motors, driver, and harmonic drive. We will calculate the profit and loss of the energy-saving method in an upcoming study.

This energy-saving effect may vary according to the pathway. We need to develop a performance index that can represent the savings effect in the overall workspace. Thereafter, the optimization of linkage parameters and type synthesis of the actuator will be performed.

\section{REFERENCES}

1. Park, C.-W., Kwon, K.-S., Kim, W.-B., Min, B.-K., Park, S.-J., et al., "Energy Consumption Reduction Technology in Manufacturing: A Selective Review of Policies, Standards, and Research," Int. J. Precis. Eng. Manuf., Vol. 10, No. 5, pp. 151-173, 2009.

2. Ahn, S.-H., "An Evaluation of Green Manufacturing Technologies Based on Research Databases,” Int. J. Precis. Eng. Manuf.-Green Tech., Vol. 1, No. 1, pp. 5-9, 2014.

3. Yoon, H.-S., Lee, J.-Y., Kim, H.-S., Kim, M.-S., Kim, E.-S., et al., "A Comparison of Energy Consumption in Bulk Forming, Subtractive, and Additive Processes: Review and Case Study," Int. J. Precis. Eng. Manuf.-Green Tech., Vol. 1, No. 3, pp. 261-279, 2014.

4. Endo, G., Yamada, H., Yajima, A., Ogata, M., and Hirose, S., "A Passive Weight Compensation Mechanism with a Non-Circular Pulley and a Spring," Proc. of the IEEE International Conference on Robotics and Automation, pp. 3843-3848, 2010.
5. Plooij, M. and Wisse, M. "A Novel Spring Mechanism to Reduce Energy Consumption of Robotic Arms," Proc. of the IEEE Intelligent Robots and Systems, pp. 2901-2908, 2012.

6. Allison, J. T., "Plant-Limited Co-Design of an Energy-Efficient Counterbalanced Robotic Manipulator," Journal of Mechanical Design, Vol. 135, No. 10, Paper No. 101003, 2013.

7. Halevi, Y., Carpanzano, E., Montalbano, G., and Koren, Y., "Minimum Energy Control of Redundant Actuation Machine Tools," CIRP Annals-Manufacturing Technology, Vol. 60, No. 1, pp. 433-436, 2011.

8. Khoukhi, A., Baron, L., and Balazinski, M., "Constrained MultiObjective Trajectory Planning of Parallel Kinematic Machines," Robotics and Computer-Integrated Manufacturing, Vol. 25, No. 4, pp. 756-769, 2009.

9. Seok, S., Wang, A., Chuah, M. Y., Otten, D., Lang, J., et al., "Design Principles for Highly Efficient Quadrupeds and Implementation on the MIT Cheetah Robot," Proc. of the IEEE International Conference on Robotics and Automation, pp. 3307-3312, 2013.

10. Lee, G., Lee, D., Jeong, J., and Kim, J., "Energy Savings of a 2-DoF Manipulator with Redundant Actuation," Proc. of the IEEE International Conference on Robotics and Automation, pp. 50765081, 2014.

11. Lee, G., Jeong, J., Park, S., Lee, D., Park, F., et al., "Minimizing Energy Consumption of Parallel Mechanisms via Redundant Actuation," IEEE/ASME Transactions on Mechatronics, DOI No. 10.1109/TMECH.2015.2401606, 2015.

12. Shin, H., Lee, S., Jeong, J. I., and Kim, J., “Antagonistic Stiffness Optimization of Redundantly Actuated Parallel Manipulators in a Predefined Workspace," IEEE/ASME Transactions on Mechatronics, Vol. 18, No. 3, pp. 1161-1169, 2013.

13. Saglia, J., Dia, J., and Caldwell, D., "Geometry and Kinematic Analysis of a Redundantly Actuated Parallel Mechanism That Eliminates Singularities and Improves Dexterity," Journal of Mechanical Design, Vol. 130, No. 12, Paper No. 124501, 2008.

14. Kennedy, C. W. and Desai, J. P., "Modeling and Control of the Mitsubishi PA-10 Robot Arm Harmonic Drive System," IEEE/ ASME Transactions on Mechatronics, Vol. 10, No. 3, pp. 263-274, 2005.

15. Harmonic Drive, "High Performance Gearheads for Servo and Stepper Motors," http://harmonicdrive.net/media/support/catalogs /pdf/gearhead-catalog.pdf (Accessed 27 February 2015)

16. Bonnans, J. F., Gilbert, J. C., Lemaréchal, C., and Sagastizábal, C. A., "Numerical Optimization: Theoretical and Practical Aspects," Springer Science \& Business Media, p. 191, 2006. 